\title{
Temperature Dependence of the Structure of Langmuir Films of Normal-Alkanes on Liquid Mercury
}

\section{Citation}

Kraack, H., B. M. Ocko, Peter S. Pershan, L. Tamam, and M. Deutsch. 2004. Temperature dependence of the structure of Langmuir films of normal-alkanes on liquid mercury. Journal of Chemical Physics 121(16): 8003-8009.

\section{Published Version}

doi:10.1063/1.1799993

\section{Permanent link}

http://nrs.harvard.edu/urn-3:HUL.InstRepos:5028195

\section{Terms of Use}

This article was downloaded from Harvard University's DASH repository, and is made available under the terms and conditions applicable to Other Posted Material, as set forth at http:// nrs.harvard.edu/urn-3:HUL.InstRepos:dash.current.terms-of-use\#LAA

\section{Share Your Story}

The Harvard community has made this article openly available.

Please share how this access benefits you. Submit a story.

\section{Accessibility}




\title{
Temperature dependence of the structure of Langmuir films of normal-alkanes on liquid mercury
}

\author{
H. Kraack \\ Department of Physics, Bar-Ilan University, Ramat-Gan 52900, Israel \\ B. M. Ocko \\ Department of Physics, Brookhaven National Laboratory, Upton, New York 11973 \\ P. S. Pershan \\ Department of Physics, Harvard University, Cambridge, Massachusetts 02138 \\ L. Tamam and M. Deutsch ${ }^{\mathrm{a}}$ \\ Department of Physics, Bar-Ilan University, Ramat-Gan 52900, Israel
}

(Received 1 April 2004; accepted 5 August 2004)

\begin{abstract}
The temperature dependent phase behavior of Langmuir films of $n$-alkanes $\left[\mathrm{CH}_{3}\left(\mathrm{CH}_{2}\right)_{n-2} \mathrm{CH}_{3}\right.$, denote $\mathrm{Cn}$ ] on mercury was studied for chain lengths $19 \leqslant n \leqslant 22$ and temperatures $15 \leqslant T$ $\leqslant 44{ }^{\circ} \mathrm{C}$, using surface tensiometry and surface $\mathrm{x}$-ray diffraction methods. In contrast with Langmuir films on water, where molecules invariably orient roughly surface normal, alkanes on mercury are always oriented surface parallel and show no long-range in-plane order at any surface pressure. A gas and several condensed phases of single, double, and triple layers of lying-down molecules are found, depending on $n$ and $T$. At high coverages, the alkanes studied here show transitions from a triple to a double to a single layer with increasing temperature. The transition temperature from a double to a single layer is found to be $\sim 5^{\circ} \mathrm{C}$, lower than the bulk rotator-to-liquid melting temperature, while the transition from a triple to a double layer is about as much below the double-to-single layer transition. Both monolayer and bulk transition temperatures show a linear increase with $n$ with identical slopes of $\sim 4.5^{\circ} \mathrm{C} \mathrm{CH}_{2}$ within the range of $n$ values addressed here. It is suggested that the film and bulk transitions are both driven by a common cause: the proliferation of gauche defects in the chain with increasing temperature. (C 2004 American
\end{abstract} Institute of Physics. [DOI: 10.1063/1.1799993]

\section{INTRODUCTION}

Langmuir films of amphiphiles on water have been studied intensively for more than a century ${ }^{1,2}$ as model systems for two-dimensional matter ${ }^{3}$ and the cell membrane ${ }^{4}$ and more recently for use in nanoscience and nanotechnology. ${ }^{5}$ These films show a large variety of structures due to a rich array of competing interactions. For example, for simple fatty acid molecules at the surface of water the nonpolar chains are attracted to each other via van der Waals forces, the polar headgroups may have competing interactions of hydrogen bonding with the water and with each other. However, the dominant effect, invariably present for organic monolayers on aqueous subphases, is the strong hydrophobic repulsion of the nonpolar hydrocarbon tail from the aqueous surface. Since until very recently all subphases employed in $\mathrm{X}$-ray studies of Langmuir films were aqueous, the molecular orientation was invariably found to be along, or slightly tilted from, the surface normal with the hydrophilic headgroup residing inside and the hydrophobic tail pointing away from the aqueous surface.

The phase diagrams of amphiphiles on water, and in particular that of fatty acid molecules on water, have been well studied by surface specific $\mathrm{x}$-ray methods ${ }^{3,6}$ ever since these

${ }^{a)}$ Electronic mail: deutsch@mail.biu.ac.il methods became available about two decades ago. ${ }^{7}$ A wealth of phases, all of them comprising roughly surface normal molecules with many different types of in-plane order, were found, depending on coverage ( $A$, the area per molecule), surface pressure $(\pi$, the difference in surface tension between a bare and a film-covered surface), temperature, $T$, and chain length, $n$.

To investigate the role of the molecule's headgroup and tail interactions with the subphase in the determination of the Langmuir film's structure, we have studied the structure of Langmuir films of fatty acids, ${ }^{8-10}$ alcohols, ${ }^{11}$ and alkanes ${ }^{12}$ on mercury, using x-ray and surface tension methods. In addition to phases with standing-up molecules, similar to those on aqueous surfaces, a variety of phases comprising surfaceparallel-oriented molecules were found, exhibiting different types of order ranging from a complete disorder to unidirectional liquid-crystal-like order to two-dimensional crystalline order. In particular, alkanes, which lack a polar headgroup, exhibit a number of lying-down phases, all of which are found to be disordered in the surface-parallel direction and ordered only in the surface-normal direction. Clearly, the high adsorption energy of the chain onto the mercury surface, $\Delta H_{\mathrm{ads}} \simeq 5.4 \mathrm{~kJ} /\left(\mathrm{mol}\right.$ of $\left.\mathrm{CH}_{2}\right),{ }^{12}$ is the main driving force for the molecular orientation, while the interchain interaction, and the methyl-methyl headgroup interaction are too weak to induce in-plane ordering. The phases were found 


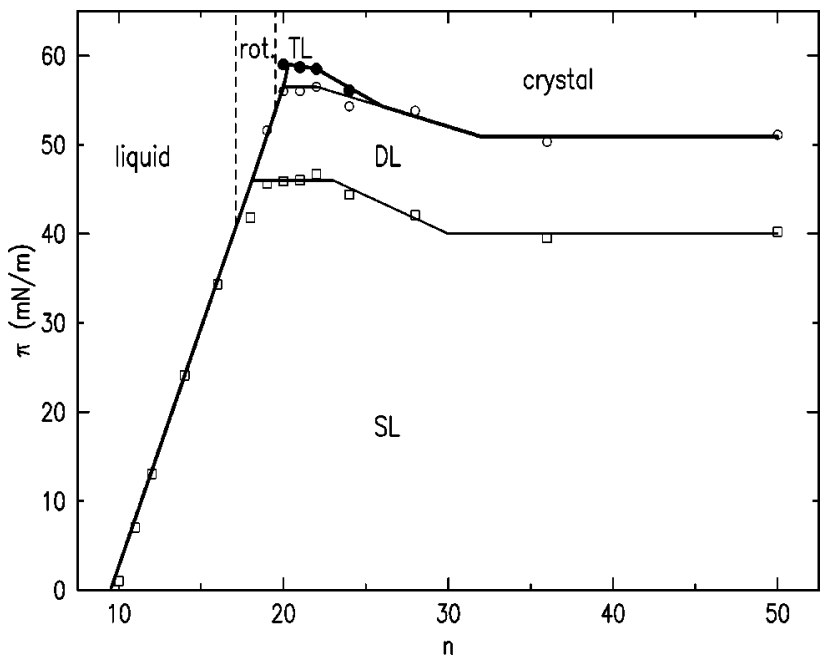

FIG. 1. The projection of the room-temperature, $T=23^{\circ} \mathrm{C}$, phase diagram of Langmuir films of alkanes on mercury onto the molecular length-surface pressure $(n, \pi)$ plane. The bold line denotes the film's collapse pressure, separating the listed 3D bulk phases above it, from the quasi-2D film phases below it. The bulk rotator phase is marked by "rot." SL, DL, and TL denote the film's single, double, and triple layer phases of lying-down molecules.

to consist of single layer (SL), double layer (DL), and triple layer (TL) of surface-parallel molecules, depending on coverage and molecular length. ${ }^{12}$ A projection of the phase diagram at room temperature, $T=23^{\circ} \mathrm{C}$, on the $(n, \pi)$ plane is given in Fig. 1, showing the lying-down phases found. The figure also shows the three-dimensional bulk phases, which are in equilibrium with the two-dimensional phases of the film at the collapse pressure, shown by a thick line in Fig. 1.

To gain a deeper understanding of the phase diagram and the various phase transitions we present here a study of the temperature dependence of the phase diagram, using x-ray and surface tension measurements for the alkane molecules $\mathrm{C} 19, \mathrm{C} 20, \mathrm{C} 21$, and C22. This is the richest part of the roomtemperature phase diagram of the Langmuir film, showing phase transitions from SL to DL to TL in the quasi-2D film and from the rotator to the crystal phases in the 3D bulk, with the liquid phase being not too far from room temperature. ${ }^{13}$

\section{EXPERIMENT}

\section{A. Langmuir trough}

We used a diffractometer-mounted Langmuir trough, allowing simultaneous $\mathrm{x}$-ray and surface tension measurements, which were carried out under inert He (x-ray) or nitrogen (surface tensiometry) atmospheres. The sample temperature is controlled by a water circulator to $\pm 0.2^{\circ} \mathrm{C}$.

For surface tension measurements the Wilhelmy plate method was used employing a mercury-amalgamated platinum plate. The film balance was based on a linear variable differential transformer, which measured the translation of the plate, hung from a leaf spring, by the force exerted on it by the surface tension.

Mercury was purchased from Merck Co. (triple distilled, 99.999\% pure) or Bethlehem Apparatus Co. (quadruple distilled 99.999 95\% pure). Alkanes were purchased from Ald- rich and were at least $98 \%$ pure. The $\mathrm{C} 22$, on which most of the measurements were made, including the temperature dependent $\mathrm{x}$-ray measurements, was obtained from FLUKA and had $99.5 \%$ purity. All materials were used as received without further purification. Standard solutions were prepared with molarities in the range of $3 \times 10^{-4}-8 \times 10^{-4}$ using HPLC grade, $99.9 \%$ pure chloroform. Films were deposited from a calibrated micropipette, through a sealable hole in the trough's enclosure.

\section{MEASUREMENT METHODS}

\section{A. Surface pressure-molecular area isotherms}

The surface pressure $\pi$ varies as a function of the Langmuir film's area per molecule $A .{ }^{2}$ The various features of the isotherm, i.e., the $\pi(A)$ curve, result from, and can be used to obtain information on, the structural changes in the Langmuir film.

As in previous experiments, ${ }^{8,9,12}$ the trough was cleaned carefully, and then its enclosure was flushed with a pure inert gas $\left(\mathrm{He}\right.$ or $\left.\mathrm{N}_{2}\right)$ for about $1 \mathrm{~h}$. Mercury was introduced from a reservoir through a capillary under the inert atmosphere. The isotherm was then measured by stepwise deposition of measured amounts of the standard solution using a calibrated micropipette, waiting after each step until an equilibrium surface pressure was reached.

\section{B. X-ray measurements}

The molecular structure of the Langmuir films was studied as a function of temperature at various points of the phase diagram using surface-specific x-ray techniques. These measurements were carried out at the Harvard/Brookhaven National Laboratory (BNL) liquid surface diffractometer at beamline X22B, NSLS, BNL, at a wavelengths of $\lambda$ $=1.571 \AA$. The trough was supported on an active vibration isolation unit, mounted on the diffractometer, an arrangement demonstrated in previous measurements ${ }^{8,12,14,15}$ to efficiently eliminate vibrational pickup from the environment.

A detailed description of the $\mathrm{x}$-ray techniques and the data analysis methods is available in the literature ${ }^{12,16,17}$ and will not be repeated here. X-ray reflectivity (XR) measurements yielded the surface-normal electron density profile and the surface roughness, while grazing incidence diffraction (GID) probed the in-plane order. As discussed below, none was found here.

\section{RESULTS AND DISCUSSION}

We discuss first the results obtained for docosane (C22) in detail, and then describe the results obtained for the other molecular lengths $19 \leqslant n \leqslant 21$.

\section{A. Docosane}

\section{Isotherms}

Isotherms of $\mathrm{C} 22$ measured for $29.0 \leqslant T \leqslant 43.6^{\circ} \mathrm{C}$ are shown in Fig. 2, with inset $B$ showing the $T=23^{\circ} \mathrm{C}$ isotherm ${ }^{12}$ and inset $C$ showing the high coverage region on a magnified scale. 


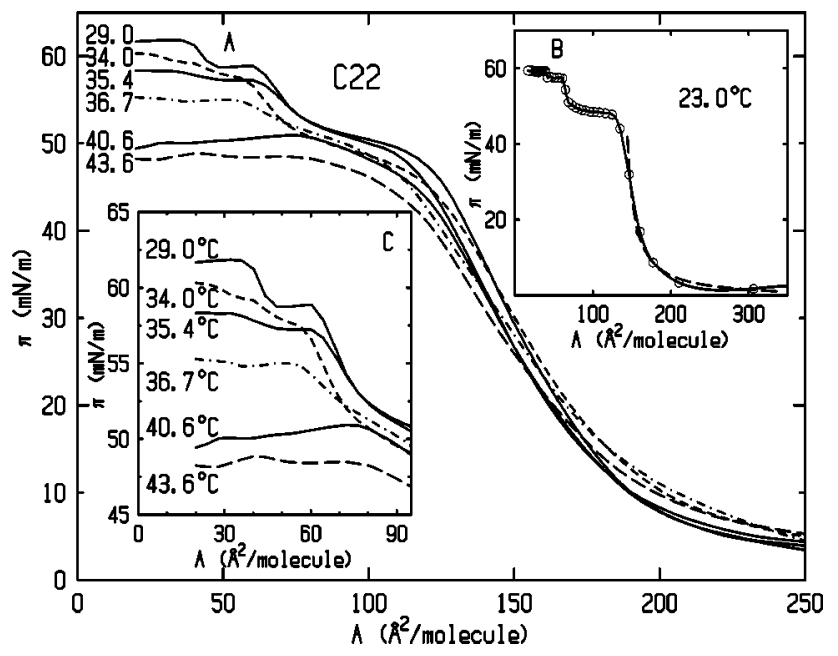

FIG. 2. (a) Isotherms of $\mathrm{C} 22$ at the indicated temperatures in degree celsius. (b) The previously-published isotherm ${ }^{12}$ at room temperature $\left(T=23^{\circ} \mathrm{C}\right)$. The dashed line is a fit by the Volmer equation for a $2 \mathrm{D}$ gas. (c) The low-A end of the isotherms on a magnified scale.

As shown in a previous room-temperature study, (Ref. 12) $\mathrm{C} 22$ at $T=23{ }^{\circ} \mathrm{C}$ forms, with decreasing $A$, successively a single, a double, and a triple layer of lying-down molecules, indicated by the appearance of three distinct steps in the isotherm. The surface pressure during the growth of the first layer follows the Volmer equation (dash line in the inset to Fig. 2) for a two-dimensional hard core gas $\pi=k T /(A$ $-A_{0}$ ) with an exclusion area $A_{0} \approx 138 \AA^{2} /$ molecule, at which coverage a closely packed single layer of lying-down molecules is obtained. ${ }^{18}$ The positions of the second and third steps in the isotherm correspond similarly to closedpacked double and triple layers. ${ }^{12}$ All phases of the $\mathrm{C} 22$ film were found to be disordered in-plane at $T=23^{\circ} \mathrm{C}$.

The isotherm at $T=29.0^{\circ} \mathrm{C}$ is very similar to that at $T$ $=23{ }^{\circ} \mathrm{C}$, showing the same three easily distinguishable plateaus at about the same coverages and roughly the same surface pressures, $\pi_{1}=48 \mathrm{mN} / \mathrm{m}, \pi_{2}=58 \mathrm{mN} / \mathrm{m}$, and $\pi_{3}$ $=61 \mathrm{mN} / \mathrm{m}$, respectively. Increasing the temperature to $T$ $=34{ }^{\circ} \mathrm{C}$ leaves most features unaltered but changes the shape of the isotherm, especially at higher coverages (lower $A$ 's). The distinction between the second and the third plateau is smoothed out. The slopes of the second and third plateaus become considerably larger than the nearly zero slopes at lower $T$. Nevertheless, even the third plateau can still be observed. However, the blurring of the shape indicates the beginning of a $T$-dependent phase transition from a three layer to a two layer phase at about this temperature. Raising the temperature further, to $T=35.4{ }^{\circ} \mathrm{C}$, causes the third plateau to almost disappear, showing only a barely distinguishable increase in $\pi$ at the onset of the third plateau, $A$ $=42 \AA^{2} /$ molecule. At $T=36.7^{\circ} \mathrm{C}$ only two plateaus are observed in the isotherm. The first plateau (extending from $A_{1}$ to $\sim 60 \mathrm{mN} / \mathrm{m}$ ) starts approximately at the same pressure as that at $T=35.4{ }^{\circ} \mathrm{C}, \pi \approx 48 \mathrm{mN} / \mathrm{m}$. The second plateaus (extending from $\sim 55 \mathrm{mN} / \mathrm{m}$ down to $\leqslant 30 \mathrm{mN} / \mathrm{m}$ ) is lower at $T=36.7^{\circ} \mathrm{C}$ by $5-6 \mathrm{mN} / \mathrm{m}$ than at $T=35.4{ }^{\circ} \mathrm{C}$, i.e., $\pi$ $\approx 55 \mathrm{mN} / \mathrm{m}$ as compared to $\pi \approx 60 \mathrm{mN} / \mathrm{m}$. This is very similar to the isotherm observed for $\mathrm{C} 19$ at room temperature. ${ }^{12}$
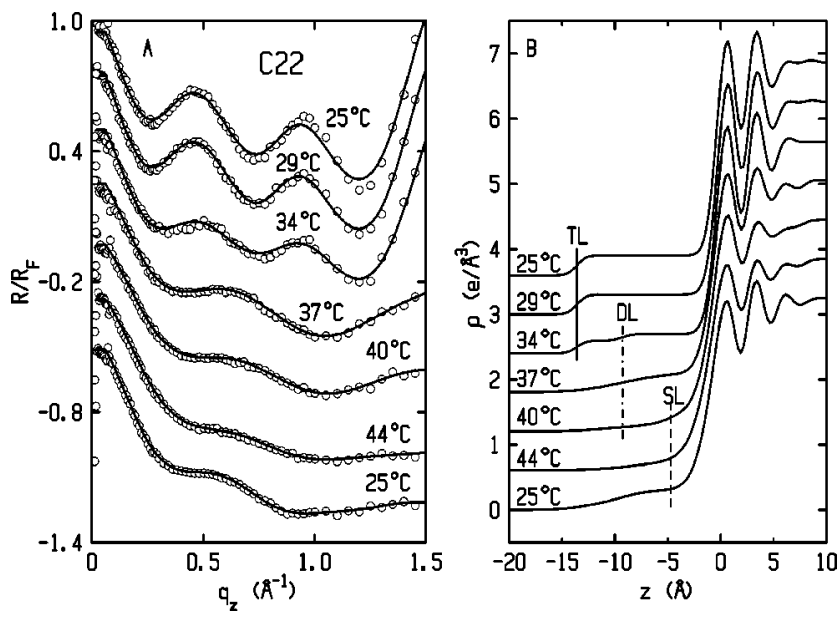

FIG. 3. (a) Measured Fresnel-normalized XR of docosane on mercury for a coverage of $40 \AA^{2} /$ molecule (open circles) at the indicated temperatures and fits (lines) by a box model. The corresponding surface pressures are listed in Table I. (b) The surface normal density profiles derived from the fits. SL, DL, and TL indicate the layer thicknesses of the single, double, and triple layers of alkanes.

In the isotherms measured at $T=40.6{ }^{\circ} \mathrm{C}$ and above only a single plateau is observed, indicating that only the SL phase is formed. Within the experimental uncertainty no systematic $T$ variation is found in either the pressure of the first plateau or in the exclusion area derived from the Volmer equation fits to the low-coverage part of the isotherms.

\section{X-ray reflectivity}

To confirm the structural hints provided by the isotherms, discussed above, we carried out temperature dependent XR measurements. Measured Fresnel-normalized XRs, $R / R_{F}$, of docosane (C22) on mercury are shown in Fig. 3(a) (open circles) as a function of increasing temperature from top to bottom, except for the lowest curve, which was measured after recooling to $T=25^{\circ} \mathrm{C}$ from the highest temperature reached, $T=44^{\circ} \mathrm{C}$. All XR were measured on the same film, with a coverage of $\sim 40 \AA^{2} /$ molecule, slightly above the nominal complete triple-layer coverage, $\sim 46 \AA^{2}$ / molecule. The stepwise film deposition was done at $25^{\circ} \mathrm{C}$ up to the onset of the third plateau in the isotherm. The temperature was then raised slowly and gradually up to $44^{\circ} \mathrm{C}$. Every few degrees the scan was interrupted, the temperature reached was held constant for $1.5 \mathrm{~h}$ to allow ample film structure equilibration, and an XR curve measured. Figure 3(a) also shows model fits (lines) to the measured $R / R_{F}$. We used the same box model and fitting procedures described previously. ${ }^{12}$ The model uses a single box of constant electron density of $\rho=0.30$ electrons $/ \AA^{3}$ for each alkane layer, and several boxes of varying electron densities to represent the layered surface ${ }^{14}$ of the mercury subphase. This value for $\rho$ was adopted after extensive set of fits with different combinations of fixed and free parameters. Changes of $\pm 5 \%$ in this value yield only negligibly small changes in the fit quality and results, and certainly have no impact on the conclusions of this paper. The density profiles derived from the fits are shown in Fig. 3(b), with $z=0$ taken at the position of the mercury-alkane interface, and the positive $z$ axis pointing 
TABLE I. Temperature $T$, surface pressure $\pi$, layer thickness $d$, phase, percentage of fill of the uppermost layer Top, the roughnesses of the alkaneair $\sigma_{\mathrm{Alk}}$, and the mercury-alkane $\sigma_{\mathrm{Hg}}$ interfaces, as obtained from fits to the $\mathrm{XR}$ curves in Fig. 3, for a $\mathrm{C} 22$ layer of a nominal coverage of $40 \AA 2 /$ molecule at room temperature. The numbers in parentheses are the estimated uncertainties in the last digit of the number quoted.

\begin{tabular}{ccccccc}
\hline \hline $\begin{array}{c}T \\
\left({ }^{\circ} \mathrm{C}\right)\end{array}$ & $\begin{array}{c}\pi \\
(\mathrm{mN} / \mathrm{m})\end{array}$ & $\begin{array}{c}d \\
(\AA)\end{array}$ & Phase & $\begin{array}{c}\text { Top } \\
(\%)\end{array}$ & $\begin{array}{c}\sigma_{\text {Alk }} \\
(\AA)\end{array}$ & $\begin{array}{c}\sigma_{\mathrm{Hg}} \\
(\AA)\end{array}$ \\
\hline 25 & $60(2)$ & $13.6(5)$ & $\mathrm{TL}$ & $100(10)$ & $0.9(3)$ & $0.9(3)$ \\
29 & $61(2)$ & $13.6(5)$ & $\mathrm{TL}$ & $100(10)$ & $1.0(3)$ & $1.0(3)$ \\
34 & $59(2)$ & $13.6(8)$ & $\mathrm{TL}$ & $65(20)$ & $1.0(4)$ & $1.0(4)$ \\
37 & $54(2)$ & $9.3(10)$ & $\mathrm{DL}$ & $100(10)$ & $3.5(10)$ & $1.4(4)$ \\
40 & $47(2)$ & $9.3(15)$ & $\mathrm{DL}$ & $40(30)$ & $3.8(15)$ & $1.6(5)$ \\
44 & $47(2)$ & $4.8(15)$ & $\mathrm{SL}$ & $100(30)$ & $5.0(15)$ & $1.9(5)$ \\
25 & $60(2)$ & $13.6(10)$ & $\mathrm{TL}$ & $25(20)$ & $1.7(10)$ & $1.7(4)$ \\
\hline \hline
\end{tabular}

into the mercury. The previously detected surface layering of mercury ${ }^{8,12,14}$ is clearly observed below the mercury-alkane interface. The quantities derived from the fit are listed in Table I.

At $25^{\circ} \mathrm{C}$ and $29^{\circ} \mathrm{C}$ the $\mathrm{XR}$ yields a layer thickness which is consistent with the interpretation of the isotherm as showing a triple layer phase TL of lying-down molecules. The peak periodicity of $\Delta q_{z} \simeq 0.46 \AA^{-1}$ in Fig. 3(a), corresponds to a layer thickness of $d=13.6 \approx 3 \times 4.7 \AA$, where $4.7 \AA$ is the thickness of a single lying-down molecule. ${ }^{12} \mathrm{At}$ $34^{\circ} \mathrm{C}$, where the third step in the isotherm was found to be very weak and almost washed out, we observe a less clear picture in the XR data as well. The oscillations in the XR curve are still observed, with peaks at the same $q_{z}$ positions, indicating that the TL phase still exists. However, they are clearly weaker than at lower temperatures, indicating that the coverage of the third layer is only partial. Indeed, the fit yields a $65 \%$ coverage for the third layer, with a full coverage for the first and second layers.

Increasing the temperature further, to $37^{\circ} \mathrm{C}$, leaves only one visible oscillation in the $\mathrm{XR}$ curve, of a longer period with a maximum at $q_{z} \approx 0.65 \AA^{-1}$. The fitted layer thickness is $9.3 \AA$ and a large surface roughness of $3.5 \AA$ is found, as compared to the roughness of $\sim 1 \AA$ at lower temperatures. The XR fit yields here a thickness equal to that of a double layer, in agreement with the conclusion derived above from the isotherm. These findings indicate that the third layer, which forms at lower temperatures at this nominal coverage, becomes unstable at this temperature. While it is likely that the molecules comprising the third layer form 3D aggregates, occupying less surface area than a monolayer, we have no observations to support this suggestion. Nevertheless, upon lowering the temperature again, the three-layer structure is restored partially, as discussed below. This indicates that the third-layer destabilization process is at least partially reversible. At even higher temperatures, the second layer also destabilizes and is gradually transformed into a single layer in equilibrium with bulk material, visible as white nonreflecting specks on the mercury surface. A fit to the measured XR curve yields a $40 \%$-full second layer at $T=40^{\circ} \mathrm{C}$ and a single layer without a second layer for $T=44{ }^{\circ} \mathrm{C}$. The roughness in both cases increased from $\sim 3.5 \AA$ for the double layer at $T=37-40{ }^{\circ} \mathrm{C}$ to about $5 \AA$ for the single layer at
$T=44{ }^{\circ} \mathrm{C}$. Recooling the film from $44{ }^{\circ} \mathrm{C}$ to $25^{\circ} \mathrm{C}$ results in the formation of a full double layer with a $25 \%$-full third layer only, rather than the fully covered third layer found at the beginning of the heating cycle. This indicates that the process is not fully reversible. The assignment of the incomplete third layer to evaporation of part of the molecules is not very likely, considering the low temperatures employed here. Finally, we wish to point out that while the fits of the XR at low temperatures converge to a unique set of parameter values, the smearing due to the large roughness at higher temperatures does not allow to obtain such well-defined fits. Several combinations of different parameter values yield reasonable fits, and a greater spread is obtained in the fitted values. We believe, however, that the cross comparison of the isotherms and the XR data makes our conclusions concerning the structure of the high- $T$ phases reasonable and reliable.

The variations in the number of layers upon heating and cooling imply that the total number of molecules included in the surface film also varies. Moreover, this variation is reversible (albeit not fully): the molecules that are removed from the surface film upon heating up, return upon cooling back down (albeit not all, as only 2.25 layers are found instead of 3). Where do these molecules go? We have no concrete experimental evidence to answer this question definitely. However, there are several more, and less, plausible possibilities, which we now discuss. We have no evidence for uncovered patches on the surface that could take up the extra material as the temperature is changed. It would be anyway difficult to justify the reversibility of the observations if this was the case. The solubility of alkanes in mercury is practically nil, so the extra material could not migrate into, and come out of, the subphase. The very low vapor pressure of the alkanes employed at these temperatures, and the reversibility, render unlikely an explanation based on molecular exchange with the vapor, considering the large volume of the trough's enclosure and possible losses of material from the vapor to the enclosure's wall, chiller plate, etc. A more plausible explanation is the formation of (nanometer-sized) 3D crystallites or multilayers, at the trough's perimeter. The perimeter would provide inhomogeneous nucleation ${ }^{19}$ sites where the extra molecules could reversibly aggregate, or disperse from, depending on the thermodynamic equilibrium between the 3D crystallites and the surface layer at any given temperature. A second possible scenario is the nucleation and dissolution of such 3D crystallites homogeneously ${ }^{19}$ at many sites across the mercury surface. The amount of material that removed and added to the surface film as the temperature is varied is small enough so that if these proto-crystallites are numerous they will occupy a very small fraction of the total area, and will not show up in the reflectivity measurements. They will be also invisible in the GID measurements, since even if they have crystalline order, their small size will render their Bragg peaks wide and low (as predicted by the Debye-Scherer formula ${ }^{20}$ ) and thus preclude their observation on top of the background scattering from the bulk and the surface film. However, as we pointed out above we have no evidence for such 3D structures either at the trough perimeter or on the surface. Clearly, this issue requires further 


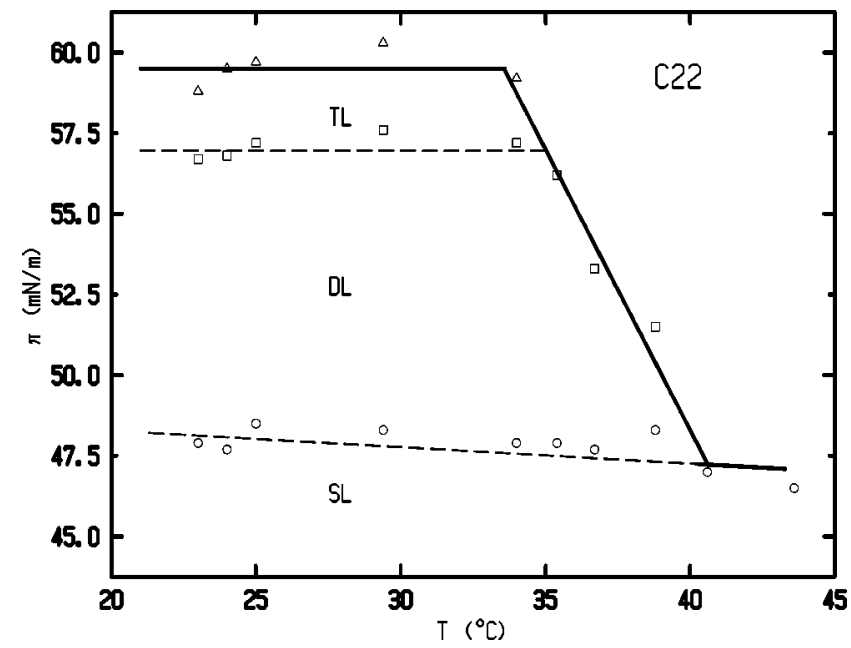

FIG. 4. The projection of the phase diagram of docosoane (C22) onto the $(T, \pi)$ plane. The bold line marks the film collapse boundary, separating the 2D film phases (TL, DL, and SL) below it from the 3D bulk phases above it. Points indicate measured values. The dashed lines mark the film's phase boundaries, and are guides to the eye only.

study before a definite conclusion can be made.

Finally, the GID measurements, carried out at each temperature, do not reveal any diffraction peaks, indicating that the Langmuir films are disordered in-plane at all $T$ for all phases. This is in agreement with the GID measurements for the alkanes studied previously at room temperature, all of which show in-plane disordered lying-down phases. ${ }^{12}$

\section{Phase diagram}

The XR and isotherm data discussed above yield the phase diagram of $\mathrm{C} 22$ on mercury in the $(T, \pi)$ plane, shown in Fig. 4.

Below $\pi \approx 47 \mathrm{mN} / \mathrm{m}$ a single layer is formed at all temperatures. Raising the pressure produces a double layer for temperatures below about $T \approx 39^{\circ} \mathrm{C}$. We find the double layer for $\pi$ between 48 and $57 \mathrm{mN} / \mathrm{m}$. For $T<34^{\circ} \mathrm{C}$ and $\pi$ $>57 \mathrm{mN} / \mathrm{m}$ a triple layer is formed. The $\pi$ range of existence of the single $(0-47 \mathrm{mN} / \mathrm{m})$, double $(47-57 \mathrm{mN} / \mathrm{m})$, and triple $(57-60 \mathrm{mN} / \mathrm{m})$ layers seems to be almost independent of $T$. Before drawing further conclusions from the present phase diagram, we would like to include in our considerations results obtained for other molecular lengths in the vicinity of docosane. These results are presented in the following section.

\section{B. Molecular length dependence}

We now summarize results obtained for C19, C20, and $\mathrm{C} 21$ in the vicinity of $\mathrm{C} 22$.

Isotherms of $\mathrm{C} 21$ for several temperatures are shown in Fig. 5 with the inset showing a magnified view of the high coverage-high pressure region. As for $\mathrm{C} 22$, at low temperatures, $22.8 \leqslant T \leqslant 30.4{ }^{\circ} \mathrm{C}$, we observe three distinct plateaus in the isotherm, indicating the formation of SL, DL, and TL phases. Similar, again, to $\mathrm{C} 22$, at the high- $T$ range around $T=30^{\circ} \mathrm{C}$, the second plateau has a relatively large slope and the third step almost disappears. In the $31.8^{\circ} \mathrm{C}$ isotherm we observe the formation of two plateaus only, at pressures of

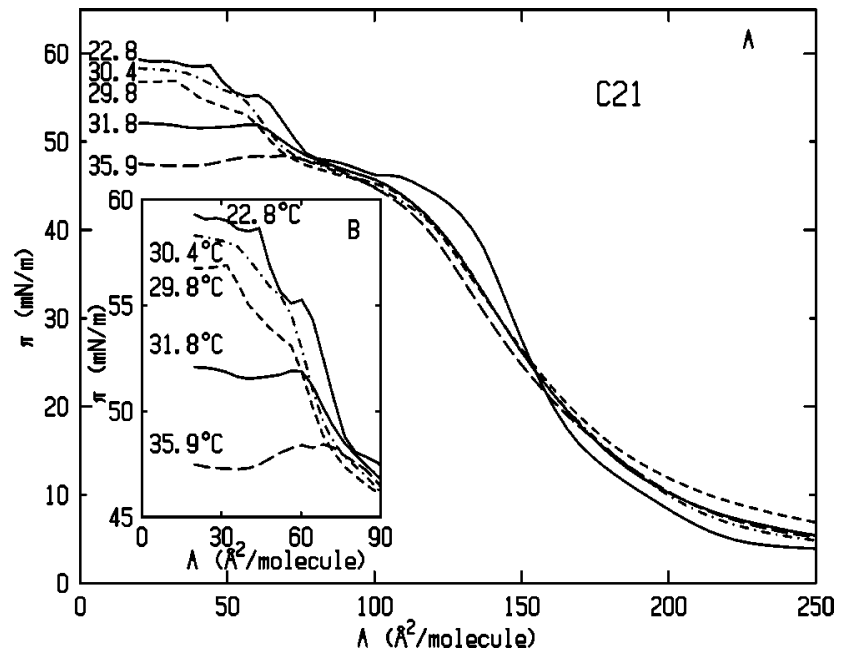

FIG. 5. Measured isotherms of $\mathrm{C} 21$ at the indicated temperatures in degree.

around $\pi_{1}=45 \mathrm{mN} / \mathrm{m}$ and $\pi_{2}=52 \mathrm{mN} / \mathrm{m}$. For even higher temperatures at $T=35.9^{\circ} \mathrm{C}$ we observe the formation of one plateau only indicating that only the SL phase occurs at this temperature.

The phase diagram derived from the isotherms is shown in Fig. 6. It is qualitatively similar to that of C22 in Fig. 4. However, unlike the phase diagram of $\mathrm{C} 22$, where the $\pi$ boundaries of the phases are horizontal, i.e., independent of temperature, here they have finite slopes, i.e., decrease roughly linearly with increasing $T$. The $T$ range of existence of the double layer is roughly the same for the two alkanes. The main differences are a downward shift in the phase transition temperatures for the lower $n$ of $4-5{ }^{\circ} \mathrm{C}$ in the $T$ boundaries of the TL and DL phases. This is reminiscent of the $n$ dependence of the phase behavior of Langmuir films of fatty acids on water, where all molecular lengths show the same universal $(T, \pi)$ phase diagram topology, once shifted up by $\sim 5^{\circ} \mathrm{C}$ per each additional $\mathrm{CH}_{2}$ group. ${ }^{3,6}$

We have also measured temperature-dependent isotherms for C19 and C20. Both sets show the same qualitative phase beahviour as that in Figs. 4 and 6 but with additional

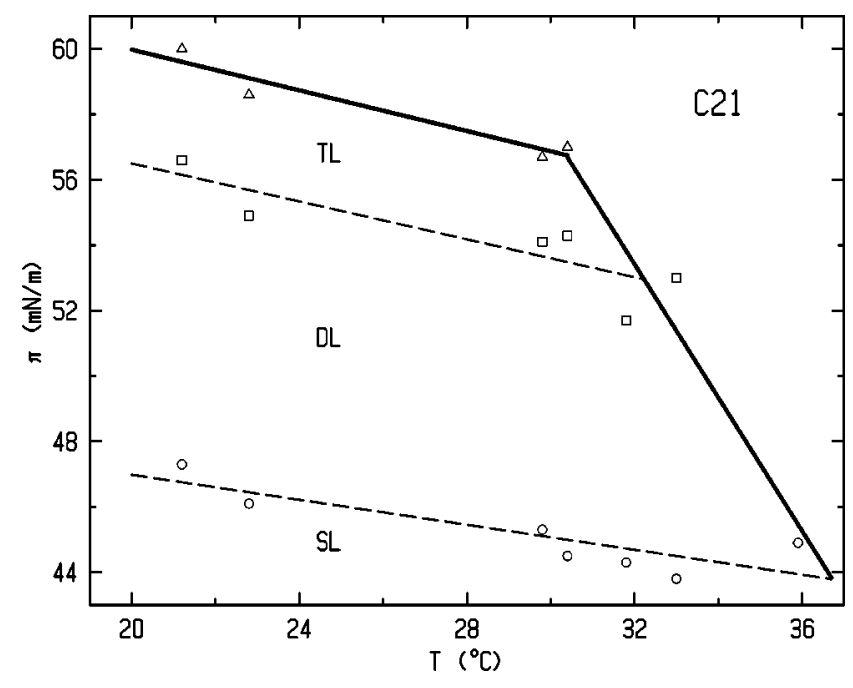

FIG. 6. Same as Fig. 4 but for eicosane (C21). 
TABLE II. Transition temperatures for the indicated molecular lengths $n$. Bulk: rotator-crystal, $T_{R-X}$, and rotator-liquid, $T_{R-L}$. Langmuir film: SLDL, $\mathrm{T}_{1-2}$ and DL-TL, $\mathrm{T}_{2-3}$.

\begin{tabular}{lcccc}
\hline \hline$n$ & $T_{R-X}$ & $T_{R-L}$ & $T_{1-2}$ & $T_{2-3}$ \\
\hline 19 & 22.0 & 32.1 & $24(1)$ & $19(1)$ \\
20 & 30.7 & 36.2 & $34(2)$ & $28(2)$ \\
21 & 31.2 & 40.1 & $35(1.5)$ & $31(1.5)$ \\
22 & 39.0 & 43.7 & $39(1)$ & $34(1)$ \\
\hline \hline
\end{tabular}

downward shifts in the transition temperatures. The transition temperatures among the various phases are listed in Table II. Fig. 7 shows the transition temperatures for the Langmuir films and in the bulk for all alkanes addressed in this study.

The bulk transition temperatures (solid lines) show the well-known linear behavior for the rotator-liquid melting transition, and the odd-even effect for the rotator-crystal transition. ${ }^{13}$ For all investigated alkanes the transition temperatures in the film, $T_{1-2}$ (squares) and $T_{2-3}$ (triangles), are roughly 5 and $10^{\circ} \mathrm{C}$, respectively, lower than the rotatorliquid melting temperatures, $T_{R-L}$. Within the experimental uncertainties, these temperatures seem also to be linear in $n$, and no odd-even effect can be claimed to occur. A linear fit is shown in dashed lines in Fig. 7. As can be observed, the $n$ slopes of both the melting temperatures of the bulk, $T_{R-L}$, and film, $T_{1-2}$ and $T_{2-3}$, are equal within their uncertainties: $d T_{R-L} / d n=(4.1 \pm 0.1){ }^{\circ} \mathrm{C} \quad$ and $\quad d T_{1-2,2-3} / d n=(4.7$ $\pm 1.2)^{\circ} \mathrm{C}$, respectively, a value close to the shift per carbon of the phase diagrams of Langmuir films of fatty acids on water, ${ }^{3,6}$ as discussed above. A plausible explanation for the identical slopes and close transition temperatures of the bulk and film transitions may be obtained by assuming that the melting of the bulk and the collapse of the molecular layers of the surface film are due to a common effect: the proliferation of gauche conformations in the molecular chains with increasing temperature. This effect distorts the regular, cigar-

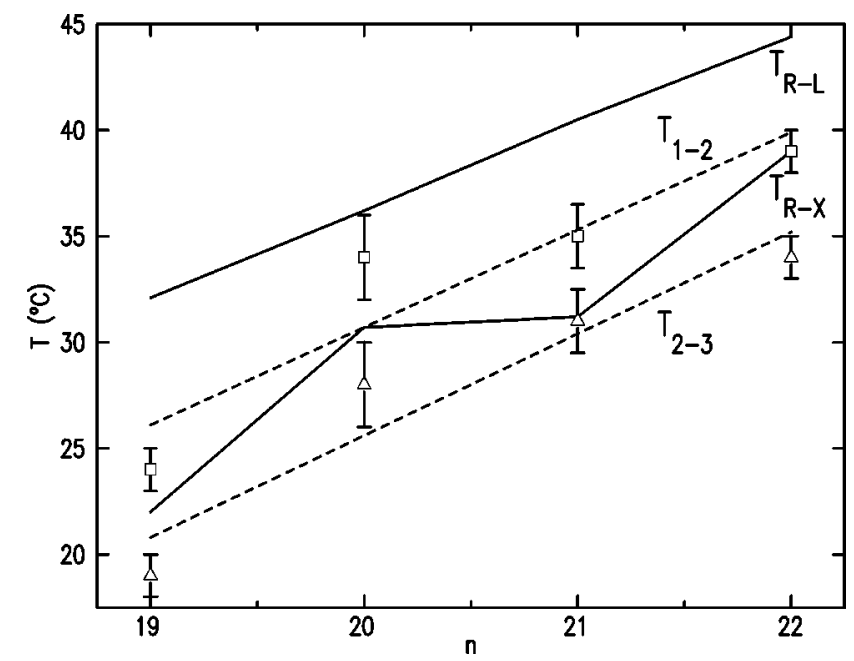

FIG. 7. Phase transition temperatures of the various phases of Langmuir films of alkanes on mercury (points) and their linear fits (dashed lines) and of bulk alkanes (solid lines), as listed in Table II. like, shape of the molecule. The distortions render a close packing of these molecules increasingly more difficult with increasing $T$, causing the bulk solid to melt and the layers in the Langmuir film to collapse at some temperature.

The only difference between the film and the bulk is the source of the order-inducing interaction, i.e., the source of the barrier which the molecular distortions have to overcome in order to cause the transition. In the bulk it is the chainchain van der Waals attraction. In the film, it is mostly the adsorption energy onto the surface. The transition temperature is determined by a competition between these orderinducing interactions and the disorder-inducing effect, the formation of gauche defects in the chains. While the chainchain attraction energy per $\mathrm{CH}_{2}$ group, $\sim 1 \mathrm{~kJ} /$ (mole of $\mathrm{CH}_{2}$ ) (Ref. 15) is smaller than the adsorption energy, $\sim 5 \mathrm{~kJ} /\left(\right.$ mole of $\left.\mathrm{CH}_{2}\right),{ }^{12}$ the difference is offset by the fact that in the bulk each molecule has six nearest neighbors, and hence a total interaction energy of 6 $\times 1 \mathrm{~kJ} /\left(\right.$ mole of $\left.\mathrm{CH}_{2}\right)$, the adsorption energy per $\mathrm{CH}_{2}$ group is counted only once for each lying-down molecule. It is therefore reasonable to expect a slightly higher transition temperature in the bulk, due to a slightly higher energy barrier, than in the film, as indeed found. Admittedly, this is only a zeroth-order approximation, and a more quantitative discussion will have to take into account smaller, though nonnegligible, contributions from, e.g., the 12 next-nearest neighbors in the bulk, the (two, for SL or three, for DL) nearest neighbors in a Hg-adsorbed layer, etc. Moreover, the balance between the disordering and ordering effects, and hence the transition temperature, should depend not on the absolute number of gauche defects but rather on their fraction of the total number of $\mathrm{CH}_{2}$ groups, i.e., the molecular length. For a longer molecule, a larger number of gauche defects, and hence a higher $T$, is required to reach the same fraction which induces a transition at a lower $T$ in a shorter chain. This implies a positive $n$ slope for the transition temperatures, and, since both surface and bulk transitions are dominated by the rate at which gauche defects are formed as $T$ is increased, also an equal $n$ slope for the transition temperatures. Both of these expectations are indeed fulfilled by the data plotted in Fig. 7, within the admittedly small number of points and the experimental uncertainties of our data. Clearly, an extension of the $n$ range of the set shown in Fig. 7 , and perhaps also an increase in its accuracy, would be of great interest in elucidating the relations between the bulk phase behavior and that of the lying-down phases of Langmuir films of alkanes on mercury.

\section{ACKNOWLEDGMENTS}

Support to M.D. by the U.S.-Israel Binational Science Foundation, Jerusalem and to P.S.P. by the U.S. DOE (Grant No. DE-FG02-88-ER45379) and the NSF (Grant No. NSFDMR-0124936) is gratefully acknowledged. The authors thank D. Vaknin (Ames) for generous advice and drawings of the trough. BNL is supported by U.S. DOE under Contract No. DE-AC02-98CH10886. 
${ }^{1}$ Lord Rayleigh, Philos. Mag. 48, 321 (1899).

${ }^{2}$ G. L. Gaines, Insoluble Monolayers at Liquid-Gas Interfaces, (Wiley, New York, 1966).

${ }^{3}$ V. M. Kaganer, H. Möhwald, and P. Dutta, Rev. Mod. Phys. 71, 779 (1999).

${ }^{4}$ S. Zheng, J. Strzalka, D. H. Jones, S. J. Opella, and J. K. Blasie, Biophys. J. 84, 2393 (2003); G. Brezesinski and H. Möhwald, Adv. Colloid Interface Sci. 100, 563 (2003).

${ }^{5}$ M. Gleiche, L. F. Chi, and H. Fuchs, Nature (London) 403, 173 (2000); A. L. S. Gamboa, E. J. M. Filipe, and P. Brogueira, Nano Lett. 2, 1083 (2002); Molecular Electronics edited by J. Jortner and M. Ratner (Balckwell, Oxford, 1997); P. Samori and J. P. Rabe, J. Phys.: Condens. Matter 14, 9955 (2002); B. A. Mantooth and P. S. Weiss, Proc. IEEE 91, 1785 (2003).

${ }^{6}$ I. R. Peterson, V. Brzezinski, R. M. Kenn, and R. Steitz, Langmuir 8, 2995 (1992).

${ }^{7}$ S. Grayer Wolf, L. Leiserowitz, M. Lahv, M. Deutsch, K. Kjaer, and J. Als-Nielsen, Nature (London) 328, 63 (1987); K. Kjaer, J. Als-Nielsen, C. A. Helm, L. A. Laxhuber, and H. Möhwald, Phys. Rev. Lett. 58, 2224 (1987); P. Dutta, J. B. Peng, B. Lin, J. B. Ketterson, M. Prakash, P. Georgopoulos, and S. Ehrlich, ibid. 58 , 2228 (1987).

${ }^{8}$ H. Kraack, B. M. Ocko, P. S. Pershan, E. Sloutskin, and M. Deutsch, Science 298, 1404 (2002).

${ }^{9}$ H. Kraack, M. Deutsch, B. M. Ocko, and P. S. Pershan, Nucl. Instrum. Methods Phys. Res. B 200, 363 (2003).

${ }^{10}$ H. Kraack, B. M. Ocko, P. S. Pershan, E. Sloutskin, L. Tamam, and M. Deutsch, Langmuir 20, 5375 (2004)
${ }^{11}$ H. Kraack, B. M. Ocko, P. S. Pershan, E. Sloutskin, L. Tamam, and M. Deutsch, Langmuir 20, 5386 (2004).

${ }^{12}$ H. Kraack, B. M. Ocko, P. S. Pershan, E. Sloutskin, and M. Deutsch, J. Chem. Phys. 119, 10339 (2003).

${ }^{13}$ E. B. Sirota and D. M. Singer, J. Chem. Phys. 101, 10873 (1994).

${ }^{14}$ O. M. Magnussen, B. M. Ocko, M. J. Regan, K. Penanen, P. S. Pershan, and M. Deutsch, Phys. Rev. Lett. 74, 4444 (1995); E. DiMasi, H. Tostman, B. M. Ocko, P. S. Pershan, and M. Deutsch, Phys. Rev. B 58, 13419 (1998).

${ }^{15}$ O. M. Magnussen, B. M. Ocko, M. Deutsch, M. J. Regan, P. S. Pershan, L. E. Berman, D. Abernathy, J. F. Legrand, and G. Grübel, Nature (London) 384, 250 (1996).

${ }^{16}$ M. Deutsch and B. M. Ocko, in Encyclopedia of Applied Physics, edited by G. L. Trigg, (VCH, New York, 1998), Vol. 23, p. 479.

${ }^{17}$ J. Als-Nielsen, D. Jacquemain, K. Kjæ r, F. Leveiller, M. Lahav, and L. Leiserowitz, Phys. Rep. 246, 252 (1994).

${ }^{18}$ The use of other equations, e.g., the $2 \mathrm{D}$ van der Waals equation of state, as well as the correspondence between the exclusion area and the geometrical area of the isotherm are discussed in Refs. 10-12. For the purpose it is employed here, i.e., to demonstrate that the first bend in the isotherm at $A_{0}$ is at a coverage commensurate with a closed-packed single layer of lyingdown molecules, the simple and least-complicated Volmer equation is sufficient.

${ }^{19}$ H. Kraack, E. B. Sirota, and M. Deutsch, J. Chem. Phys. 112, 6873 (2000)

${ }^{20}$ A. Guinier, X-Ray Diffraction (Freeman, San Francisco, 1963), Chap. 5.3. 
The Journal of Chemical Physics is copyrighted by the American Institute of Physics (AIP). Redistribution of journal material is subject to the AIP online journal license and/or AIP copyright. For more information, see http:/ojps.aip.org/jcpo/jcpcr/jsp Copyright of Journal of Chemical Physics is the property of American Institute of Physics and its content may not be copied or emailed to multiple sites or posted to a listserv without the copyright holder's express written permission. However, users may print, download, or email articles for individual use. 